TRANSACTIONS OF THE

AMERICAN MATHEMATICAL SOCIETY

Volume 361, Number 10, October 2009, Pages 5163-5175

S 0002-9947(09)04736-9

Article electronically published on April 7, 2009

\title{
A CHARACTERIZATION OF COMPACT PERTURBATIONS OF TOEPLITZ OPERATORS
}

\author{
JINGBO XIA
}

\begin{abstract}
Let $X$ be a bounded operator on the Hardy space $H^{2}$ of the unit circle. It has been a longstanding problem to determine whether the condition that $T_{\bar{u}} X T_{u}-X$ is compact for every inner function $u$ implies that $X$ is a compact perturbation of a Toeplitz operator. We show that the answer is affirmative.
\end{abstract}

\section{INTRODUCTION}

Let $\mathbf{T}$ be the unit circle $\{z \in \mathbf{C}:|z|=1\}$ and let $d m$ be the Lebesgue measure on $\mathbf{T}$ with the normalization $m(\mathbf{T})=1$. We will simply write $L^{p}$ for $L^{p}(\mathbf{T}, d m)$, $1 \leq p \leq \infty$. Recall that the Hardy space $H^{2}$ is the closure of the linear span of $\left\{z^{n}: n=0,1,2, \ldots\right\}$ in $L^{2}$, where $z$ denotes the coordinate function on $\mathbf{T}$. Let $P: L^{2} \rightarrow H^{2}$ be the orthogonal projection. For any $f \in L^{\infty}$, the Toeplitz operator $T_{f}$ is defined by the formula

$$
T_{f}=P M_{f} \mid H^{2} .
$$

There is a very simple characterization of Toeplitz operators due to Brown and Halmos [1; namely, a bounded operator $B$ on $H^{2}$ is a Toeplitz operator if and only if it satisfies the equation

$$
T_{\bar{z}} B T_{z}=B
$$

Given this fact, it is natural to ask, how does one characterize compact perturbations of Toeplitz operators? In other words, how does one characterize operators of the form $T_{f}+K$, where $f \in L^{\infty}$ and $K$ is a compact operator on $H^{2}$ ? An answer was proposed in Douglas's influential textbook [3]. Observe that if $u$ is an inner function, then $T_{\bar{u}} T_{f} T_{u}=T_{|u|^{2} f}=T_{f}$ for every $f \in L^{\infty}$. Thus if $X$ is an operator of the form $X=T_{f}+K$, where $f \in L^{\infty}$ and $K$ is compact, then for each inner function $u$ the difference

$$
T_{\bar{u}} X T_{u}-X
$$

is necessarily a compact operator. Douglas asks, does the converse hold true? That is, if $X$ is a bounded operator on $H^{2}$ which has the property that

$$
T_{\bar{u}} X T_{u}-X \text { is compact for every inner function } u \text {, }
$$

does it follow that $X=T_{f}+K$ for some $f \in L^{\infty}$ and compact $K$ ? This is one of the famous "double-asterisk" problems in [3] (see pages xii and 207).

Received by the editors August 20, 2007.

2000 Mathematics Subject Classification. Primary 47A55, 47B35.

This work was supported by National Science Foundation grant DMS-0456448.

(C)2009 American Mathematical Society 
In the years since the publication of [3], the closest to the solution of this problem was a result due to Davidson [2]. Davidson showed that if $X$ has the property that $\left[X, T_{h}\right]$ is compact for every $h \in H^{\infty}$, then $X=T_{f}+K$, where $f \in L^{\infty}$ and $K$ is compact. This result has the implication that the essential commutant of the full Toeplitz algebra is $\mathcal{T}(\mathrm{QC})$.

By a well-known theorem of Marshall [8, the set of inner functions generates $H^{\infty}$ as a Banach algebra. Therefore the condition that $\left[X, T_{h}\right]$ is compact for every $h \in H^{\infty}$ is equivalent to the condition that $\left[X, T_{u}\right]$ is compact for every inner function $u$. What makes Davidson's result slightly weaker than the solution of Douglas's original problem is the fact that the condition $\left[T_{f}, T_{h}\right]$ is compact for every $h \in H^{\infty}$ implies $f \in H^{\infty}+C(\mathbf{T})\left[9\right.$. Consequently, the condition that $\left[X, T_{u}\right]$ is compact for every inner function $u$ is stronger than the condition that $T_{\bar{u}} X T_{u}-X$ is compact for every inner function $u$.

Thus when the second edition of Douglas's book was published in 1998, this " $T_{\bar{u}} X T_{u}-X$ " problem still carried the double asterisk 4, page 184], signifying that a solution was still being sought.

We will settle the issue in this paper.

Theorem 1. Let $X$ be a bounded operator on $H^{2}$. If $T_{\bar{u}} X T_{u}-X$ is compact for every inner function $u$, then $X=T_{f}+K$, where $f \in L^{\infty}$ and $K$ is a compact operator on $\mathrm{H}^{2}$.

So how do we prove this theorem? Naively, one would start with the weak limit $X_{0}$ of a subsequence of $\left\{T_{\bar{z}^{n}} X T_{z^{n}}\right\}$ and try to prove that $X-X_{0}$ is compact. After all, if $X$ has the desired form $T_{f}+K$, then $X-X_{0}$ recovers $K$. The problem with this naive approach is that we do not see how to prove the compactness of $X-X_{0}$. In some sense, our approach is just a slight modification of the naive approach. Instead of the inner functions $z^{n}$, we use $w_{n}$ defined in (1.1) below. Although one can think of $w_{n}$ as a modification of $z^{n}$, we will see that this modification makes a qualitative difference. In Section 1 we establish an easy proposition which reduces the proof of Theorem 1 to the proof of the compactness of a very specific operator constructed using $w_{n}$. This sets the course for the rest of the proof. The next step, which takes up Section 2 , is to extract a more user-friendly " $\epsilon-\delta$ " condition from the condition that $T_{\bar{u}} X T_{u}-X$ is compact for every inner function $u$. In Section 3 we use the " $\epsilon-\delta$ " condition and other relevant facts to prove the compactness of that specific operator. We conclude the paper with a remark in Section 4.

\section{A sequence of Blaschke products}

For the rest of the paper we fix a positive number $0<\alpha<1$. For each $n \in \mathbf{N}$, define

$$
w_{n}(z)=\frac{\alpha-z^{n}}{1-\alpha z^{n}} .
$$

The zeros of $w_{n}$ are precisely the $n$-th roots of $\alpha$.

Lemma 2. If $K$ is a compact operator on $H^{2}$, then

$$
\lim _{n \rightarrow \infty}\left\|T_{\bar{w}_{n}} K T_{w_{n}}-\alpha^{2} K\right\|=0 .
$$

Proof. Since $w_{n}(z)=\alpha+\left(\alpha^{2}-1\right) z^{n}\left(1-\alpha z^{n}\right)^{-1}$, we can rewrite it in the form

$$
w_{n}(z)=\alpha+z^{n} f_{n}(z)
$$


with $f_{n}(z)=\left(\alpha^{2}-1\right)\left(1-\alpha z^{n}\right)^{-1}$. Therefore

$$
T_{\bar{w}_{n}} K T_{w_{n}}-\alpha^{2} K=\alpha T_{\bar{f}_{n}} T_{\bar{z}^{n}} K+\alpha K T_{z^{n}} T_{f_{n}}+T_{\bar{f}_{n}} T_{\bar{z}^{n}} K T_{z^{n}} T_{f_{n}} .
$$

For a compact operator $K$, it is well known that $\left\|T_{\bar{z}^{n}} K\right\| \rightarrow 0$ as $n \rightarrow \infty$. Thus $\left\|K T_{z^{n}}\right\|=\left\|T_{\bar{z}^{n}} K^{*}\right\|$ also tends to 0 as $n \rightarrow \infty$. Since $\left\|f_{n}\right\|_{\infty} \leq 1+\alpha$, the lemma follows.

We would like to remind the reader of the elementary fact that for a bounded operator $A$ on $H^{2}, T_{\bar{z}} A T_{z}-A$ is compact if and only if $\left[A, T_{\varphi}\right]$ is compact for every $\varphi \in C(\mathbf{T})$.

Proposition 3. Let $Y$ be a bounded operator on $H^{2}$ such that the commutator $\left[Y, T_{\varphi}\right]$ is compact for every $\varphi \in C(\mathbf{T})$. Let $n(1)<\ldots<n(\nu)<\ldots$ be a strictly increasing sequence of natural numbers such that the weak limit

$$
Z=\mathrm{w}-\lim _{\nu \rightarrow \infty} T_{\bar{w}_{n(\nu)}} Y T_{w_{n(\nu)}}
$$

exists. Then $\left[Z, T_{\varphi}\right]$ is compact for every $\varphi \in C(\mathbf{T})$. Moreover, there exists an $f \in L^{\infty}$ such that

$$
Y=T_{f}+\left(1-\alpha^{2}\right)^{-1}(Y-Z)
$$

Proof. We have

$$
\begin{aligned}
T_{\bar{z}} Z T_{z}-Z & =\mathrm{w}-\lim _{\nu \rightarrow \infty}\left(T_{\bar{z}} T_{\bar{w}_{n(\nu)}} Y T_{w_{n(\nu)}} T_{z}-T_{\bar{w}_{n(\nu)}} Y T_{w_{n(\nu)}}\right) \\
& =\mathrm{w}-\lim _{\nu \rightarrow \infty} T_{\bar{w}_{n(\nu)}}\left(T_{\bar{z}} Y T_{z}-Y\right) T_{w_{n(\nu)}} \\
& =\alpha^{2}\left(T_{\bar{z}} Y T_{z}-Y\right)
\end{aligned}
$$

where the last $=$ follows from the compactness of $T_{\bar{z}} Y T_{z}-Y$ and Lemma 2 . Hence $T_{\bar{z}} Z T_{z}-Z$ is compact, which implies that $\left[Z, T_{\varphi}\right]$ is compact for every $\varphi \in C(\mathbf{T})$.

The above shows that the operator $B=Y-\alpha^{-2} Z$ satisfies the equation $T_{\bar{z}} B T_{z}-$ $B=0$. By the Brown-Halmos criterion, $B$ is a Toeplitz operator. That is, there is a $g \in L^{\infty}$ such that $Y-\alpha^{-2} Z=B=T_{g}$. By elementary algebra, if we set $f=\left(1-\alpha^{-2}\right)^{-1} g$, then (1.2) holds.

\section{An " $\epsilon-\delta "$ CONDITION}

In this paper the only inner functions we need are Blaschke products. Furthermore, most of the work involves finite Blaschke products, using the infinite Blaschke product only once. Given a finite Blaschke product

$$
b(z)=e^{i \theta} \prod_{j=1}^{n} \frac{a_{j}-z}{1-\bar{a}_{j} z},
$$

where $n \in \mathbf{N}, \theta \in \mathbf{R}$, and $\left|a_{j}\right|<1$ for $j=1, \ldots, n$, we define

$$
R(b)=\sum_{j=1}^{n}\left(1-\left|a_{j}\right|\right) .
$$

As we will see, $R(b)$ is an important ingredient in the proof of Theorem 1.

The quantity $R(b)$ has appeared in the literature before. In [5, Section 4.2], this quantity was involved in a characterization of the non-cyclic vectors of the backward shift (i.e., $T_{\bar{z}}$ ). For a finite Blaschke product $b$, if its zeros are simple, then it is an interpolating Blaschke product. In the case that $b$ is an interpolating 
Blaschke product, there is a Carleson measure naturally associated with it [6, page 286], and $R(b)$ is just the total mass of this Carleson measure. (The author thanks the referee for suggesting the inclusion of this background information.)

For the finite Blaschke product $w_{n}$ defined by (1.1), we have

$$
R\left(w_{n}\right)=n\left(1-\alpha^{1 / n}\right) .
$$

The point here is that, as $n \rightarrow \infty, R\left(w_{n}\right)$ has a finite limit $\log \left(\alpha^{-1}\right)$. In contrast,

$$
R\left(z^{n}\right)=n .
$$

For the rest of the paper, let $\mathcal{F}$ denote the collection of finite Blaschke products.

Lemma 4. Let $\left\{b_{k}\right\}$ be a sequence in $\mathcal{F}$ such that

$$
\lim _{k \rightarrow \infty} R\left(b_{k}\right)=0 .
$$

(i) Then for every bounded operator $A$ on $H^{2}$ we have the strong convergence

$$
\text { s- } \lim _{k \rightarrow \infty}\left(T_{\bar{b}_{k}} A T_{b_{k}}-A\right)=0 .
$$

(ii) For every bounded operator $A$ on $H^{2}$ and every $b \in \mathcal{F}$ we have

$$
\lim _{k \rightarrow \infty}\left\{\left\|T_{\bar{b}_{k}} A T_{b_{k}}-A\right\|-\left\|T_{\bar{b}}\left(T_{\bar{b}_{k}} A T_{b_{k}}-A\right) T_{b}\right\|\right\}=0 .
$$

Proof. Let $b \in \mathcal{F}$. Note that if $\tau$ is a complex number in $\mathbf{T}$ such that $\tau b(0) \in[0,1)$, then $\tau b(0)=\left|a_{1}\right| \ldots\left|a_{n}\right|$, where $a_{1}, \ldots, a_{n}$ are the zeros of $b$, counting multiplicity. Since $\left|a_{j}\right|<1$ for every $j$, it is elementary that $1-\left|a_{1}\right| \ldots\left|a_{n}\right| \leq\left(1-\left|a_{1}\right|\right)+\ldots+$ $\left(1-\left|a_{n}\right|\right)$. In other words, the inequality

$$
1-\tau b(0) \leq R(b)
$$

holds if $\tau \in \mathbf{T}$ is such that $\tau b(0) \in[0,1)$.

Now let $\left\{b_{k}\right\} \subset \mathcal{F}$ and suppose that (2.1) holds. For each $k \in \mathbf{N}$, let $\tau_{k} \in \mathbf{T}$ be such that $\tau_{k} b_{k}(0) \in[0,1)$. Then by $(2.2)$ and $(2.1)$ we have

$$
\lim _{k \rightarrow \infty}\left\|1-\tau_{k} b_{k}\right\|^{2}=\lim _{k \rightarrow \infty}\left(2-2 \operatorname{Re}\left\langle\tau_{k} b_{k}, 1\right\rangle\right)=2 \lim _{k \rightarrow \infty}\left(1-\tau_{k} b_{k}(0)\right)=0 .
$$

As usual, let $M_{f}$ denote the operator of multiplication by $f$ on $L^{2}$. Since $L^{\infty}$ is dense in $L^{2}$, the above gives us the strong convergence

$$
\text { s- } \lim _{k \rightarrow \infty} M_{\tau_{k} b_{k}}=1
$$

on the Hilbert space $L^{2}$. Since $T_{\bar{\tau}_{k} \bar{b}_{k}} A T_{\tau_{k} b_{k}}=T_{\bar{b}_{k}} A T_{b_{k}}$, this proves (i).

To prove (ii), let $b \in \mathcal{F}$ also be given. Let $E$ and $F$ be the orthogonal projections from $H^{2}$ onto the range of $T_{b}$ and the kernel of $T_{\bar{b}}$, respectively. Since $b$ is a finite Blaschke product, we have $\operatorname{rank}(F)<\infty$. Thus, applying (i) to $A^{*}$ as well as to $A$, we have

$$
\lim _{k \rightarrow \infty}\left\|F\left(T_{\bar{b}_{k}} A T_{b_{k}}-A\right)\right\|=0=\lim _{k \rightarrow \infty}\left\|\left(T_{\bar{b}_{k}} A T_{b_{k}}-A\right) F\right\| .
$$

On the other hand, $T_{b}$ is an isometry that maps $H^{2}$ onto $E H^{2}$. Therefore

$$
\left\|T_{\bar{b}}\left(T_{\bar{b}_{k}} A T_{b_{k}}-A\right) T_{b}\right\|=\left\|E\left(T_{\bar{b}_{k}} A T_{b_{k}}-A\right) E\right\| .
$$


Consequently

$$
\begin{aligned}
\left\|T_{\bar{b}}\left(T_{\bar{b}_{k}} A T_{b_{k}}-A\right) T_{b}\right\| \leq & \left\|T_{\bar{b}_{k}} A T_{b_{k}}-A\right\| \\
\leq & \left\|T_{\bar{b}}\left(T_{\bar{b}_{k}} A T_{b_{k}}-A\right) T_{b}\right\|+\left\|F\left(T_{\bar{b}_{k}} A T_{b_{k}}-A\right) E\right\| \\
& +\left\|E\left(T_{\bar{b}_{k}} A T_{b_{k}}-A\right) F\right\|+\left\|F\left(T_{\bar{b}_{k}} A T_{b_{k}}-A\right) F\right\| .
\end{aligned}
$$

Combining this with (2.3), (ii) follows.

Lemma 5. Let $X$ be a bounded operator on $H^{2}$ such that $T_{\bar{u}} X T_{u}-X$ is compact for every inner function $u$. Then for every $\epsilon>0$, there exists a $\delta=\delta(\epsilon)>0$ such that

$$
\left\|T_{\bar{b}} X T_{b}-X\right\| \leq \epsilon
$$

for every $b \in \mathcal{F}$ with $R(b) \leq \delta$.

Proof. Suppose the contrary. Then there would be an $\epsilon>0$ such that for every $k \epsilon$ $\mathbf{N}$, there is a $b_{k} \in \mathcal{F}$ satisfying the conditions

$$
R\left(b_{k}\right) \leq 2^{-k}
$$

and

$$
\left\|T_{\bar{b}_{k}} X T_{b_{k}}-X\right\| \geq \epsilon .
$$

Replacing $b_{k}$ by $\tau_{k} b_{k}$ with an appropriate $\tau_{k} \in \mathbf{T}$ if necessary, we may assume that

$$
b_{k}(0) \in[0,1)
$$

for every $k \in \mathbf{N}$. We will complete the proof by showing that such a sequence $\left\{b_{k}\right\}$ leads to a contradiction.

We claim that there exists a strictly increasing sequence of natural numbers $k(1)<k(2)<\ldots<k(j)<\ldots$ and a family of finite-rank orthogonal projections $\left\{F_{j}: j \in \mathbf{N}\right\}$ which are mutually orthogonal, i.e., $F_{j} F_{j^{\prime}}=0=F_{j^{\prime}} F_{j}$ if $j \neq j^{\prime}$, such that

$$
\begin{aligned}
\left\|F_{j+1} T_{\bar{v}_{j}}\left(T_{\bar{b}_{k(j+1)}} X T_{b_{k(j+1)}}-X\right) T_{v_{j}} F_{j+1}\right\| & \geq \epsilon / 2 \\
\left\|\left(1-F_{j+1}\right) T_{\bar{v}_{j}}\left(T_{\bar{b}_{k(j+1)}} X T_{b_{k(j+1)}}-X\right) T_{v_{j}}\right\| & \leq 2^{-j} \quad \text { and } \\
\left\|T_{\bar{v}_{j}}\left(T_{\bar{b}_{k(j+1)}} X T_{b_{k(j+1)}}-X\right) T_{v_{j}}\left(1-F_{j+1}\right)\right\| & \leq 2^{-j}
\end{aligned}
$$

for every $j \in \mathbf{N}$, where

$$
v_{j}=\prod_{\nu=1}^{j} b_{k(\nu)} .
$$

We will find these sequences inductively. We begin with an arbitrary $k(1) \geq 1$ and an arbitrary orthogonal projection $F_{1}$ with $\operatorname{rank}\left(F_{1}\right)<\infty$. Suppose that $j \geq 1$ and that we have selected $k(1)<\ldots<k(j)$ and finite-rank orthogonal projections $F_{1}, \ldots, F_{j}$ which are mutually orthogonal. To select $k(j+1)$ and $F_{j+1}$, we set $G_{j}=F_{1}+\ldots+F_{j}$. Since $\operatorname{rank}\left(G_{j}\right)<\infty$, it follows from Lemma 4(i) that

$$
\lim _{k \rightarrow \infty}\left(\left\|G_{j} T_{\bar{v}_{j}}\left(T_{\bar{b}_{k}} X T_{b_{k}}-X\right) T_{v_{j}}\right\|+\left\|T_{\bar{v}_{j}}\left(T_{\bar{b}_{k}} X T_{b_{k}}-X\right) T_{v_{j}} G_{j}\right\|\right)=0 .
$$


By this limit and by the combination of Lemma 4(ii) and (2.5), there exists a $k(j+1)>k(j)$ such that

$$
\begin{aligned}
\left\|T_{\bar{v}_{j}}\left(T_{\bar{b}_{k(j+1)}} X T_{b_{k(j+1)}}-X\right) T_{v_{j}}\right\| & \geq 3 \epsilon / 4 \\
\left\|G_{j} T_{\bar{v}_{j}}\left(T_{\bar{b}_{k(j+1)}} X T_{b_{k(j+1)}}-X\right) T_{v_{j}}\right\| & \leq \min \left\{2^{-j-1}, \epsilon / 12\right\} \quad \text { and } \\
\left\|T_{\bar{v}_{j}}\left(T_{\bar{b}_{k(j+1)}} X T_{b_{k(j+1)}}-X\right) T_{v_{j}} G_{j}\right\| & \leq \min \left\{2^{-j-1}, \epsilon / 12\right\} .
\end{aligned}
$$

The operator $T_{\bar{b}_{k(j+1)}} X T_{b_{k(j+1)}}-X$ is compact by assumption. Thus there exists a finite-rank orthogonal projection $G_{j+1}$ such that $G_{j+1} \geq G_{j}$ and such that

$$
\begin{aligned}
\left\|G_{j+1} T_{\bar{v}_{j}}\left(T_{\bar{b}_{k(j+1)}} X T_{b_{k(j+1)}}-X\right) T_{v_{j}} G_{j+1}\right\| & \geq 2 \epsilon / 3, \\
\left\|\left(1-G_{j+1}\right) T_{\bar{v}_{j}}\left(T_{\bar{b}_{k(j+1)}} X T_{b_{k(j+1)}}-X\right) T_{v_{j}}\right\| & \leq 2^{-j-1} \quad \text { and } \\
\left\|T_{\bar{v}_{j}}\left(T_{\bar{b}_{k(j+1)}} X T_{b_{k(j+1)}}-X\right) T_{v_{j}}\left(1-G_{j+1}\right)\right\| & \leq 2^{-j-1} .
\end{aligned}
$$

Let $F_{j+1}=G_{j+1}-G_{j}$. The requirement $G_{j+1} \geq G_{j}$ ensures that $F_{j+1}$ is an orthogonal projection, and $F_{j+1}$ is obviously orthogonal to $F_{1}, \ldots, F_{j}$. Now $(2.7)$ follows from the combination of (2.13), (2.11) and (2.12). Similarly, (2.8) follows from (2.11) and (2.14), and (2.9) follows from (2.12) and (2.15). Thus we have inductively produced sequences $\{k(j)\}$ and $\left\{F_{j}\right\}$ such that (2.7-2.9) hold for every $j \geq 1$.

For each $j \geq 1$, define

$$
K_{j}=T_{\bar{v}_{j}}\left(T_{\bar{b}_{k(j+1)}} X T_{b_{k(j+1)}}-X\right) T_{v_{j}}-F_{j+1} T_{\bar{v}_{j}}\left(T_{\bar{b}_{k(j+1)}} X T_{b_{k(j+1)}}-X\right) T_{v_{j}} F_{j+1},
$$

which is, of course, a compact operator. By (2.8) and (2.9), $\left\|K_{j}\right\| \leq 2^{-j}+2^{-j}=$ $2^{-j+1}$. Thus the operator

$$
K=\sum_{j=1}^{\infty} K_{j}
$$

is compact. Because the $F_{j}$ 's are mutually orthogonal, the sum

$$
S=\sum_{j=1}^{\infty} F_{j+1} T_{\bar{v}_{j}}\left(T_{\bar{b}_{k(j+1)}} X T_{b_{k(j+1)}}-X\right) T_{v_{j}} F_{j+1}
$$

converges in the strong operator topology. If $\left\{f_{j+1}\right\}$ is a sequence of unit vectors such that $f_{j+1} \in F_{j+1} H^{2}$ for every $j$, then the sequence $\left\{f_{j+1}\right\}$ weakly converges to 0 . Thus (2.7) guarantees that $S$ is not a compact operator.

Using induction, it is easy to see that for every $n \in \mathbf{N}$,

$$
T_{\bar{v}_{n+1}} X T_{v_{n+1}}-X=\left(T_{\bar{b}_{k(1)}} X T_{b_{k(1)}}-X\right)+\sum_{j=1}^{n} T_{\bar{v}_{j}}\left(T_{\bar{b}_{k(j+1)}} X T_{b_{k(j+1)}}-X\right) T_{v_{j}} .
$$

From the preceding paragraph it is obvious that we have the strong convergence

$$
\text { S- } \lim _{n \rightarrow \infty} \sum_{j=1}^{n} T_{\bar{v}_{j}}\left(T_{\bar{b}_{k(j+1)}} X T_{b_{k(j+1)}}-X\right) T_{v_{j}}=S+K .
$$

Conditions (2.4) and (2.6) ensure the existence of the infinite Blaschke product

$$
v=\prod_{j=1}^{\infty} b_{k(j)}
$$


Furthermore, these conditions guarantee the strong operator convergence

$$
\text { s- } \lim _{n \rightarrow \infty} M_{v_{n}}=M_{v}
$$

on the Hilbert space $L^{2}$. Combining this convergence with (2.16) and (2.17), we now have

$$
T_{\bar{v}} X T_{v}-X=\left(T_{\bar{b}_{k(1)}} X T_{b_{k(1)}}-X\right)+S+K
$$

Since $K$ is compact while $S$ is not, this contradicts the assumption on $X$.

\section{Estimates}

For each $n \in \mathbf{N}$, let $\Phi_{n}$ denote the collection of the $n$-th roots of $\alpha$. Given any subset $G$ of $\mathbf{T}$, we also define

$$
\Phi_{n}(G)=\left\{\omega \in \Phi_{n}: \omega /|\omega| \in G\right\}
$$

and

$$
w_{n, G}(z)=\prod_{\omega \in \Phi_{n}(G)} \frac{\omega-z}{1-\bar{\omega} z}
$$

In the case $\Phi_{n}(G)=\emptyset, w_{n, G}$ is understood to mean the constant function 1 .

Lemma 6. There exists a constant $0<C<\infty$ such that the inequality

$$
\left|\frac{r e^{i \mu}-e^{i s}}{1-r e^{-i \mu} e^{i s}}-\frac{r e^{i \mu}-e^{i t}}{1-r e^{-i \mu} e^{i t}}\right| \leq C \frac{(1-r)|s-t|}{|\mu-s||\mu-t|}
$$

holds for all real numbers $r, \mu, s, t$ satisfying the conditions $0<r<1,0<|\mu-s| \leq$ $3 \pi / 2$ and $0<|\mu-t| \leq 3 \pi / 2$.

Proof. Writing $\tau_{1}=e^{i(s-\mu)}$ and $\tau_{2}=e^{i(t-\mu)}$, we have

$$
\left|\frac{r e^{i \mu}-e^{i s}}{1-r e^{-i \mu} e^{i s}}-\frac{r e^{i \mu}-e^{i t}}{1-r e^{-i \mu} e^{i t}}\right|=\frac{\left(1-r^{2}\right)\left|\tau_{2}-\tau_{1}\right|}{\left|1-r \tau_{1}\right|\left|1-r \tau_{2}\right|} \leq 8 \frac{(1-r)\left|1-\tau_{1} \bar{\tau}_{2}\right|}{\left|1-\tau_{1}\right|\left|1-\tau_{2}\right|},
$$

where the $\leq$ follows from the elementary fact that $2|1-r \tau| \geq|1-\tau|$ for all $\tau \in$ $\mathbf{T}$ and $0<r<1$. To estimate the numerator above, note that there is a constant $0<C_{1}<\infty$ such that $\left|1-e^{i x}\right| \leq C_{1}|x|$ for all $x \in \mathbf{R}$. For the denominator, note that the value

$$
c_{2}=\inf _{0<y \leq 3 \pi / 2}\left|\frac{1-e^{i y}}{y}\right|
$$

is greater than 0 . This completes the proof.

For each pair of $k \in \mathbf{N}$ and $j \in \mathbf{Z}$ we define the closed arc

$$
I_{k, j}=\left\{e^{2 \pi i x}: 2^{-k} j \leq x \leq 2^{-k}(j+1)\right\}
$$

in $\mathbf{T}$. Then, of course, $I_{k, j+2^{k}}=I_{k, j}$.

Lemma 7. There exists a constant $0<C_{7}<\infty$ such that the following estimate holds. Let $k \geq 3, j \in \mathbf{Z}$ and $M \geq 3$. Let $G=\mathbf{T} \backslash\left\{\bigcup_{\nu=j-M}^{j+M} I_{k, \nu}\right\}$. Then

$$
\left|w_{n, G}(z)-w_{n, G}\left(z^{\prime}\right)\right| \leq \frac{C_{7}}{M}
$$

for all $z, z^{\prime} \in I_{k, j-1} \cup I_{k, j} \cup I_{k, j+1}$ and $n \geq 2^{k}$. 
Proof. Given $z, z^{\prime} \in I_{k, j-1} \cup I_{k, j} \cup I_{k, j+1}$, we can write $z=e^{2 \pi i x}$ and $z^{\prime}=e^{2 \pi i y}$ with $x, y \in\left[2^{-k}(j-1), 2^{-k}(j+2)\right]$. If $\omega \in \Phi_{n}(G)$, then we can write $\omega=\alpha^{1 / n} e^{2 \pi i(\ell / n)}$ with an $\ell \in \mathbf{Z}$ such that

$$
\frac{M}{2^{k}} \leq\left|\frac{j}{2^{k}}-\frac{\ell}{n}\right| \leq \frac{1}{2}
$$

Since $M \geq 3$, we have $|x-(\ell / n)| \geq\left|\left(j / 2^{k}\right)-(\ell / n)\right|-\left|x-\left(j / 2^{k}\right)\right| \geq(1 / 3) \mid\left(j / 2^{k}\right)-$ $(\ell / n) \mid$. Similarly, $|y-(\ell / n)| \geq(1 / 3)\left|\left(j / 2^{k}\right)-(\ell / n)\right|$. Applying Lemma 5 , we find that

$$
\begin{aligned}
\left|\frac{\omega-z}{1-\bar{\omega} z}-\frac{\omega-z^{\prime}}{1-\bar{\omega} z^{\prime}}\right| & \leq C \frac{\left(1-\alpha^{1 / n}\right)|x-y|}{2 \pi|x-(\ell / n)||y-(\ell / n)|} \leq 9 C \frac{2^{-k}\left(1-\alpha^{1 / n}\right)}{\left|\left(j / 2^{k}\right)-(\ell / n)\right|^{2}} \\
& \leq \frac{9 C C^{\prime}}{2^{k} n\left|\left(j / 2^{k}\right)-(\ell / n)\right|^{2}}
\end{aligned}
$$

where $C^{\prime}=\sup _{\nu \geq 1} \nu\left(1-\alpha^{1 / \nu}\right)$. Note that for any $\tau_{1}, \ldots, \tau_{m}, \tau_{1}^{\prime}, \ldots, \tau_{m}^{\prime} \in \mathbf{T}$, we have

$$
\left|\tau_{1} \ldots \tau_{m}-\tau_{1}^{\prime} \ldots \tau_{m}^{\prime}\right| \leq\left|\tau_{1}-\tau_{1}^{\prime}\right|+\ldots+\left|\tau_{m}-\tau_{m}^{\prime}\right|
$$

Hence, writing $C_{1}$ for $9 C C^{\prime}$, from (3.1) and (3.2) we deduce

$$
\left|w_{n, G}(z)-w_{n, G}\left(z^{\prime}\right)\right| \leq \sum_{\left|\left(j / 2^{k}\right)-(\ell / n)\right| \geq 2^{-k} M} \frac{C_{1}}{2^{k} n\left|\left(j / 2^{k}\right)-(\ell / n)\right|^{2}}
$$

in the event $\Phi_{n}(G) \neq \emptyset$. Splitting the set $\left\{\ell \in \mathbf{Z}:\left|\left(j / 2^{k}\right)-(\ell / n)\right| \geq 2^{-k} M\right\}$ as the union of $\left\{\ell \in \mathbf{Z}:(\ell / n)-\left(j / 2^{k}\right) \geq 2^{-k} M\right\}$ and $\left\{\ell \in \mathbf{Z}:(\ell / n)-\left(j / 2^{k}\right) \leq-2^{-k} M\right\}$, we see that

$$
\left|w_{n, G}(z)-w_{n, G}\left(z^{\prime}\right)\right| \leq 2 \sum_{\nu=0}^{\infty} \frac{C_{1}}{2^{k} n\left\{\left(M / 2^{k}\right)+(\nu / n)\right\}^{2}} .
$$

Let $n \geq 2^{k}$. Then $\left(n / 2^{k}\right) M \geq M \geq 3$. Thus

$$
\sum_{\nu=0}^{\infty} \frac{1}{2^{k} n\left\{\left(M / 2^{k}\right)+(\nu / n)\right\}^{2}}=\frac{n}{2^{k}} \sum_{\nu=0}^{\infty} \frac{1}{\left\{\left(n / 2^{k}\right) M+\nu\right\}^{2}} \leq \frac{n}{2^{k}} \cdot \frac{C_{2}}{\left(n / 2^{k}\right) M}=\frac{C_{2}}{M}
$$

under our assumptions. Substituting this in (3.3), the lemma follows.

Suppose that $A$ is a bounded operator on a Hilbert space $\mathcal{H}$. Recall that the essential norm of $A$ is defined by the formula

$$
\|A\|_{\mathcal{Q}}=\inf \{\|A+K\|: K \text { is compact on } \mathcal{H}\} .
$$

Proposition 8. Let $A$ be a bounded operator on $H^{2}$ such that $\left[A, T_{\varphi}\right]$ is compact for every $\varphi \in C(\mathbf{T})$. Then there exists a $\gamma \in \mathbf{T}$ such that the inequality

$$
\left\|T_{\xi} A\right\|_{\mathcal{Q}} \geq(1 / 2)\|A\|_{\mathcal{Q}}
$$

holds for every $\xi \in C(\mathbf{T})$ satisfying the condition $\xi(\gamma)=1$.

Proof. We use Douglas's localization technique [3. Let $\mathcal{K}$ denote the collection of compact operators on $H^{2}$. Let $\mathcal{T}(C(\mathbf{T}))$ be the $C^{*}$-algebra generated by $\left\{T_{\varphi}: \varphi \in\right.$ $C(\mathbf{T})\}$. Let $\mathcal{D}$ be the $C^{*}$-algebra generated by $A$ and $\mathcal{T}(C(\mathbf{T}))$. Finally, for each $\tau \in \mathbf{T}$, let $\mathcal{I}_{\tau}$ denote the ideal in $\mathcal{D}$ generated by $\left\{T_{\varphi}: \varphi(\tau)=0\right.$ and $\left.\varphi \in C(\mathbf{T})\right\}$. By the well-known properties of $\mathcal{T}(C(\mathbf{T}))$, we have $\mathcal{I}_{\tau} \supset \mathcal{K}$ for every $\tau \in \mathbf{T}$. 
Since $\mathcal{T}(C(\mathbf{T})) / \mathcal{K}$ is contained in the center of $\mathcal{D} / \mathcal{K}$, it follows from 3 , Theorem 7.47] that $\bigcap_{\tau \in \mathbf{T}}\left(\mathcal{I}_{\tau} / \mathcal{K}\right)=\{0\}$. Therefore $\bigcap_{\tau \in \mathbf{T}} \mathcal{I}_{\tau}=\mathcal{K}$ and, by an elementary $C^{*}$-algebraic argument,

$$
\sup _{\tau \in \mathbf{T}}\left\|A+\mathcal{I}_{\tau}\right\|=\|A+\mathcal{K}\|=\|A\|_{\mathcal{Q}} .
$$

Hence there exists a $\gamma \in \mathbf{T}$ such that

$$
\left\|A+\mathcal{I}_{\gamma}\right\| \geq(1 / 2)\|A\|_{\mathcal{Q}} .
$$

Suppose that $\xi \in C(\mathbf{T})$ and that $\xi(\gamma)=1$. Then $A-T_{\xi} A \in \mathcal{I}_{\gamma}$. Therefore

$$
\left\|T_{\xi} A+\mathcal{I}_{\gamma}\right\|=\left\|A+\mathcal{I}_{\gamma}\right\| \geq(1 / 2)\|A\|_{\mathcal{Q}}
$$

Since $\mathcal{I}_{\gamma} \supset \mathcal{K}$, this implies (3.4).

We have now arrived at the main step in the proof of Theorem 1.

Proposition 9. Suppose that $Y$ is a bounded operator on $H^{2}$ satisfing the following two conditions:

(a) For every $\varphi \in C(\mathbf{T})$, the commutator $\left[Y, T_{\varphi}\right]$ is compact.

(b) For every $\epsilon>0$, there exists a $\delta=\delta(\epsilon)>0$ such that $\left\|T_{\bar{b}} Y T_{b}-Y\right\| \leq \epsilon$ for every $b \in \mathcal{F}$ with $R(b) \leq \delta$.

Then there exist an $f \in L^{\infty}$ and a compact operator $K$ on $H^{2}$ such that

$$
Y=T_{f}+K \text {. }
$$

Proof. There exists a strictly increasing sequence $n(1)<\ldots<n(\nu)<\ldots$ of natural numbers such that the weak limit

$$
Z=\mathrm{w}-\lim _{\nu \rightarrow \infty} T_{\bar{w}_{n(\nu)}} Y T_{w_{n(\nu)}}
$$

exists. To complete the proof, according to Proposition 3, it suffices to show that the operator $Y-Z$ is compact. To prove this, we assume the contrary, i.e.,

$$
\|Y-Z\|_{\mathcal{Q}}=c
$$

for some $c>0$. We will show that this leads to a contradiction.

By Proposition $3,\left[Z, T_{\varphi}\right]$ is compact for every $\varphi \in C(\mathbf{T})$. Thus Proposition 8 can be applied to $Y-Z$. By Proposition 8 and (3.5), there is a $\gamma \in \mathbf{T}$ such that

$$
\left\|T_{\xi}(Y-Z)\right\|_{\mathcal{Q}} \geq c / 2 \quad \text { if } \xi(\gamma)=1, \xi \in C(\mathbf{T}) .
$$

We set

$$
a=\frac{c}{32\|Y\|} .
$$

Since $c \leq 2\|Y\|$, we have $a<1$. For each $k \geq 1$, there is a $j(k) \in\left\{1, \ldots, 2^{k}\right\}$ such that

$$
\gamma \in I_{k, j(k)}=\left\{e^{2 \pi i x}: 2^{-k} j(k) \leq x \leq 2^{-k}(j(k)+1)\right\} .
$$

For each $k \geq 1$, define the open arc

$$
S_{k}=\left\{e^{2 \pi i x}: 2^{-k}(j(k)-(1 / 2))<x<2^{-k}(j(k)+1+(1 / 2))\right\} .
$$

By a standard construction, for each $k \geq 1$ there is a function $\psi_{k}$ satisfying the following conditions:

(1) $\psi_{k} \in C^{\infty}(\mathbf{T})$.

(2) $\psi_{k}(\gamma)=1$.

(3) $a^{2} \leq \psi_{k} \leq 1$ on $\mathbf{T}$.

(4) $\psi_{k}=a^{2}$ on $\mathbf{T} \backslash S_{k}$. 
By (3.6) and (2), we have

$$
\left\|T_{\psi_{k}}(Y-Z)\right\|_{\mathcal{Q}} \geq c / 2
$$

for every $k \geq 1$. By (1) and (3), the function $\log \psi_{k}$ belongs to $C^{\infty}(\mathbf{T})$. Hence the function

$$
h_{k}(z)=\frac{1}{2} \int_{\mathbf{T}} \frac{\tau+z}{\tau-z} \log \left(\psi_{k}(\tau)\right) d m(\tau),
$$

which is analytic on $D=\{z:|z|<1\}$, has a continuous extension to the closed unit disc $\bar{D}$. Define

$$
g_{k}=e^{h_{k}}
$$

Then $g_{k}$ is analytic on $D$, continuous on $\bar{D}$, and

$$
\left|g_{k}\right|^{2}=\psi_{k} \quad \text { on } \mathbf{T} \text {. }
$$

Hence $T_{\psi_{k}}=T_{\bar{g}_{k}} T_{g_{k}}$ and we can rewrite (3.7) as

$$
\left\|T_{\bar{g}_{k}} T_{g_{k}}(Y-Z)\right\|_{\mathcal{Q}} \geq c / 2 .
$$

Since $g_{k}$ is continuous on $\mathbf{T}$, the commutator $\left[T_{g_{k}}, Y-Z\right]$ is compact. Therefore we can replace $T_{\bar{g}_{k}} T_{g_{k}}(Y-Z)$ by $T_{\bar{g}_{k}}(Y-Z) T_{g_{k}}$ in (3.8) to obtain

$$
\left\|T_{\bar{g}_{k}}(Y-Z) T_{g_{k}}\right\|_{\mathcal{Q}} \geq c / 2 \text {. }
$$

Since the operator norm dominates the essential norm, this gives us

$$
\left\|T_{\bar{g}_{k}} Y T_{g_{k}}-T_{\bar{g}_{k}} Z T_{g_{k}}\right\|=\left\|T_{\bar{g}_{k}}(Y-Z) T_{g_{k}}\right\| \geq c / 2 .
$$

Since $T_{\bar{g}_{k}} Z T_{g_{k}}$ is the weak limit of $\left\{T_{\bar{g}_{k}} T_{\bar{w}_{n(\nu)}} Y T_{w_{n(\nu)}} T_{g_{k}}\right\}$ and $g_{k}$ is analytic, we now have

$$
\liminf _{\nu \rightarrow \infty}\left\|T_{\bar{g}_{k}} Y T_{g_{k}}-T_{\bar{w}_{n(\nu)} \bar{g}_{k}} Y T_{w_{n(\nu)} g_{k}}\right\| \geq c / 2
$$

for every $k \geq 1$.

Next we will replace the $w_{n(\nu)}$ in (3.9) with $b_{k, \nu} \in \mathcal{F}$ such that $R\left(b_{k, \nu}\right)$ is small. To do this we first pick an integer $M \geq 3$ such that

$$
\frac{C_{7}}{M} \leq a=\frac{c}{32\|Y\|}
$$

where $C_{7}$ is the constant provided by Lemma 7 . For each $k \geq 3$, define

$$
G_{k}=\mathbf{T} \backslash\left\{\bigcup_{j=j(k)-M}^{j(k)+M} I_{k, j}\right\} .
$$

For each $k \geq 3$, pick an arbitrary $z_{k} \in S_{k}$. Since

$$
S_{k} \subset I_{k, j(k)-1} \cup I_{k, j(k)} \cup I_{k, j(k)+1},
$$

it follows from Lemma 7 that if $n(\nu) \geq 2^{k}$, then

$$
\left|w_{n(\nu), G_{k}}(z)-w_{n(\nu), G_{k}}\left(z_{k}\right)\right| \leq C_{7} / M \leq a \quad \text { for all } z \in S_{k} .
$$

For $k \geq 3$ and $\nu \geq 1$, define

$$
\tau_{k, \nu}=w_{n(\nu), G_{k}}\left(z_{k}\right)
$$

and

Then (3.10) translates to

$$
b_{k, \nu}=w_{n(\nu)} / w_{n(\nu), G_{k}} .
$$

$$
\left|w_{n(\nu)}(z)-\tau_{k, \nu} b_{k, \nu}(z)\right| \leq a \quad \text { for all } z \in S_{k}
$$


provided that $\nu$ satisfies the condition $n(\nu) \geq 2^{k}$. Combining this inequality with (3), (4) and the fact that $\left|g_{k}\right|=\psi_{k}^{1 / 2}$ on $\mathbf{T}$, we conclude that

$$
\left\|w_{n(\nu)} g_{k}-\tau_{k, \nu} b_{k, \nu} g_{k}\right\|_{\infty} \leq 2 a
$$

if $\nu$ satisfies the condition $n(\nu) \geq 2^{k}$. Since $a=(32\|Y\|)^{-1} c$ and since $\bar{\tau}_{k, \nu} \tau_{k, \nu}=1$, it follows from the above inequality and (3.9) that

$$
\liminf _{\nu \rightarrow \infty}\left\|T_{\bar{g}_{k}} Y T_{g_{k}}-T_{\bar{b}_{k, \nu} \bar{g}_{k}} Y T_{b_{k, \nu} g_{k}}\right\| \geq c / 4
$$

for every $k \geq 3$. Since $T_{b_{k, \nu} g_{k}}=T_{b_{k, \nu}} T_{g_{k}}$, this means

$$
\liminf _{\nu \rightarrow \infty}\left\|T_{\bar{g}_{k}}\left(Y-T_{\bar{b}_{k, \nu}} Y T_{b_{k, \nu}}\right) T_{g_{k}}\right\| \geq c / 4 \text {. }
$$

Finally, because $\left|g_{k}\right|=\psi_{k}^{1 / 2} \leq 1$ on $\mathbf{T}$, we conclude that

$$
\liminf _{\nu \rightarrow \infty}\left\|Y-T_{\bar{b}_{k, \nu}} Y T_{b_{k, \nu}}\right\| \geq c / 4
$$

for every $k \geq 3$.

On the other hand, condition (b) tells us that there is a $\delta_{0}>0$ such that

$$
\left\|Y-T_{\bar{b}} Y T_{b}\right\| \leq c / 8 \quad \text { for every } \quad b \in \mathcal{F} \text { with } R(b) \leq \delta_{0} .
$$

We will show that there is an $N \geq 3$ such that

$$
\limsup _{\nu \rightarrow \infty} R\left(b_{k, \nu}\right) \leq \delta_{0} / 2 \quad \text { for every } k \geq N
$$

Clearly, (3.11) and (3.13) together contradict (3.12), proving the compactness of $Y-Z$. To prove $(3.13)$, consider the arc

$$
H_{k}=\bigcup_{j=j(k)-M}^{j(k)+M} I_{k, j}=\left\{e^{2 \pi i x}: 2^{-k}(j(k)-M) \leq x \leq 2^{-k}(j(k)+M+1)\right\}
$$

$k \geq 3$. By the definition of $w_{n(\nu), G_{k}}$, the zeros of $b_{k, \nu}$ are the $n(\nu)$-th roots of $\alpha$ contained in $\Phi_{n(\nu)}\left(H_{k}\right)$. Hence

$$
\begin{aligned}
R\left(b_{k, \nu}\right) & =\sum_{\omega \in \Phi_{n(\nu)}\left(H_{k}\right)}(1-|\omega|)=\left(1-\alpha^{1 / n(\nu)}\right) \operatorname{card}\left(\Phi_{n(\nu)}\left(H_{k}\right)\right) \\
& \leq \frac{C^{\prime}}{n(\nu)} \operatorname{card}\left(\Phi_{n(\nu)}\left(H_{k}\right)\right)
\end{aligned}
$$

where $C^{\prime}=\sup _{n \geq 1} n\left(1-\alpha^{1 / n}\right)$. To estimate $\operatorname{card}\left(\Phi_{n(\nu)}\left(H_{k}\right)\right)$, note that for any $n \in \mathbf{N}, \ell \in \mathbf{Z}$ and $-\infty<x<y<\infty$, we have $\ell / n \in[x, y]$ if and only if $\ell \in[n x, n y]$. Therefore

$\operatorname{card}\left(\Phi_{n(\nu)}\left(H_{k}\right)\right) \leq 1+n(\nu)\left\{2^{-k}(j(k)+M+1)-2^{-k}(j(k)-M)\right\}=1+n(\nu) \frac{2 M+1}{2^{k}}$.

Consequently

$$
R\left(b_{k, \nu}\right) \leq C^{\prime}\left(\frac{1}{n(\nu)}+\frac{2 M+1}{2^{k}}\right)
$$

Thus if we pick $N \geq 3$ such that $2^{-N} C^{\prime}(2 M+1) \leq \delta_{0} / 2$, then (3.13) holds. This completes the proof of the proposition.

Proof of Theorem 1. This follows immediately from Lemma 5 and Proposition 9. 


\section{A Remark on Proposition 9}

Conditions (a) and (b) in Proposition 9 are also necessary for $Y$ to have the form $T_{f}+K$, where $f \in L^{\infty}$ and $K$ is compact. The necessity of (a) is obvious. The necessity of (b) certainly follows from Lemma 5 , but it is equally certain that we do not need to quote Lemma 5 to obtain the necessity of (b). It is an easy exercise to show directly that if $K$ is compact, then for any $\epsilon>0$, there is a $\delta=\delta(\epsilon)>0$ such that $\left\|T_{\bar{b}} K T_{b}-K\right\| \leq \epsilon$ for every $b \in \mathcal{F}$ with $R(b) \leq \delta$. We leave this exercise to the interested reader.

In any case, we have the following alternate characterization of compact perturbations of Toeplitz operators on $H^{2}$ : A bounded operator $Y$ on $H^{2}$ has the form $T_{f}+K$, where $f \in L^{\infty}$ and $K$ is compact, if and only if it satisfies both condition (a) and condition (b) in Proposition 9. The key word here is "both". Any inquisitive mind may wonder, is condition (b) alone a characterization of compact perturbations of Toeplitz operators on $H^{2}$ ? In other words, it is natural to ask

Question 10. Does condition (b) in Proposition 9 imply condition (a)?

The answer is negative. In the remainder of the section, we give an example of an operator which satisfies (b) but not (a). This is in fact the same operator $E$ that we used in [10] for a different purpose. Let us recall the relevant construction from [10].

Let $\mathcal{E}$ be the norm closure of $\operatorname{span}\left\{z^{2^{k}}: k \in \mathbf{N}\right\}$ in $H^{2}$. If $\psi \in \mathcal{E}$, then its Taylor expansion on the unit disc $D$ is a lacunary power series. The sparsity of the powers dictates the multiplicative behavior of the functions in $\mathcal{E}$, and the multiplicative behavior of these functions results in the norm inequality (4.1) below. Write $\|\cdot\|_{r}$ for the $L^{r}$-norm.

Let $E: H^{2} \rightarrow \mathcal{E}$ be the orthogonal projection. It is obvious that

$$
\left[T_{z}, E\right] z^{2^{k}}=z^{1+2^{k}}
$$

$k \in \mathbf{N}$. Thus the operator $\left[T_{z}, E\right]$ is not compact. Consequently $E$ does not satisfy condition (a). Next we show that $E$ satisfies condition (b).

Consider $k, \ell, k^{\prime}, \ell^{\prime} \in \mathbf{N}$ satisfying the conditions $k \leq \ell$ and $k^{\prime} \leq \ell^{\prime}$. For such $k, \ell, k^{\prime}, \ell^{\prime}$, it is obvious that

$$
2^{k}+2^{\ell} \neq 2^{k^{\prime}}+2^{\ell^{\prime}} \quad \text { if either } \ell \neq \ell^{\prime} \text { or } k \neq k^{\prime} .
$$

Thus for any polynomial $p(z)=\sum_{k=1}^{N} c_{k} z^{2^{k}}$ in $\mathcal{E}$, we have

$$
\|p\|_{4}^{4}=\left\langle p^{2}, p^{2}\right\rangle=\sum_{k=1}^{N}\left|c_{k}^{2}\right|^{2}+\sum_{1 \leq k<\ell \leq N} 4\left|c_{k} c_{\ell}\right|^{2} \leq 2\left(\sum_{k=1}^{N}\left|c_{k}\right|^{2}\right)^{2}=2\|p\|_{2}^{4} .
$$

Let $f \in L^{\infty}$. If $q$ is a polynomial in $H^{2}$, then $E q$ is a polynomial in $\mathcal{E}$. Therefore

$$
\left\|T_{f} E q\right\|_{2} \leq\|f E q\|_{2} \leq\|E q\|_{4}\|f\|_{4} \leq 2^{1 / 4}\|E q\|_{2}\|f\|_{4} \leq 2^{1 / 4}\|q\|_{2}\|f\|_{4} .
$$

Since polynomials are dense in $H^{2}$, it follows that $\left\|T_{f} E\right\| \leq 2^{1 / 4}\|f\|_{4}$ and $\left\|E T_{f}\right\|=$ $\left\|T_{\bar{f}} E\right\| \leq 2^{1 / 4}\|\bar{f}\|_{4}$ for every $f \in L^{\infty}$. Hence

$$
\left\|\left[E, T_{f}\right]\right\| \leq 2^{5 / 4}\|f\|_{4} \quad \text { for every } f \in L^{\infty} .
$$


Now let $b \in \mathcal{F}$. Let $\tau \in \mathbf{T}$ be such that $\tau b(0) \in[0,1)$. We have

$$
\begin{aligned}
\left\|\left[E, T_{b}\right]\right\| & =\left\|\left[E, T_{b-\bar{\tau}}\right]\right\| \leq 2^{5 / 4}\|b-\bar{\tau}\|_{4} \leq 2^{5 / 4}\|b-\bar{\tau}\|_{\infty}^{1 / 2}\|b-\bar{\tau}\|_{2}^{1 / 2} \\
& \leq 2^{5 / 4} \cdot 2^{1 / 2} \cdot\{2-2 \tau b(0)\}^{1 / 4} \leq 4\{R(b)\}^{1 / 4},
\end{aligned}
$$

where the last $\leq$ follows from $(2.2)$. For $b \in \mathcal{F}$, we have $\left\|T_{\bar{b}} E T_{b}-E\right\|=\left\|T_{\bar{b}}\left[E, T_{b}\right]\right\|$ $\leq\left\|\left[E, T_{b}\right]\right\|$. Thus the operator $E$ satisfies condition (b) in Proposition 9.

\section{REFERENCES}

1. A. Brown and P. Halmos, Algebraic properties of Toeplitz operators, J. Reine Angew. Math. 213 (1963/1964), 89-102. MR0160136 (28:3350)

2. K. Davidson, On operators commuting with Toeplitz operators modulo the compact operators, J. Funct. Anal. 24 (1977), 291-302. MR0454715 (56:12963)

3. R. Douglas, Banach algebra techniques in operator theory, Pure and Applied Mathematics, 49, Academic Press, New York-London, 1972. MR0361893 (50:14335)

4. R. Douglas, Banach algebra techniques in operator theory, 2nd ed., Graduate Texts in Mathematics, 179, Springer-Verlag, New York, 1998. MR1634900 (99c:47001)

5. R. Douglas, H. Shapiro and A. Shields, Cyclic vectors and invariant subspaces for the backward shift operator, Ann. Inst. Fourier (Grenoble) 20 (1970), 37-76. MR0270196 (42:5088)

6. J. Garnett, Bounded analytic functions, Academic Press, New York-London, 1981. MR628971 (83g:30037)

7. B. Johnson and S. Parrott, Operators commuting with a von Neumann algebra modulo the set of compact operators, J. Funct. Anal. 11 (1972), 39-61. MR0341119 (49:5869)

8. D. Marshall, Blaschke products generate $H^{\infty}$, Bull. Amer. Math. Soc. 82 (1976), 494-496. MR 0402054 (53:5877)

9. D. Sarason, On products of Toeplitz operators, Acta Sci. Math. (Szeged) 35 (1973), 7-12. MR0331109 (48:9443)

10. J. Xia, On the essential commutant of $\mathcal{T}(\mathrm{QC})$, Trans. Amer. Math. Soc. 360 (2008), 10891102. MR 2346484

Department of Mathematics, State University of New York at Buffalo, Buffalo, NEW YorK 14260

E-mail address: jxia@acsu.buffalo.edu 